\title{
Study of Thermosiphon Cooling Scheme for the Production Solenoid of the Mu2e Experiment at Fermilab
}

\author{
N. Dhanaraj ${ }^{\mathrm{a}}$, V. Kashikhin ${ }^{\mathrm{a}}$, T. Peterson ${ }^{\mathrm{a}}$, V. Pronskikh ${ }^{\mathrm{a}}$, T. Nicol $^{\mathrm{a}}$ \\ ${ }^{a}$ Fermi National Accelerator Laboratory, P. O. Box 500, Batavia, IL 60510 USA
}

\begin{abstract}
A thermosiphon cooling scheme is envisioned for the Production Solenoid of the Mu2e experiment at Fermi National Accelerator Laboratory. The anticipated heat loads in the solenoid will be presented as well as the cooling scheme design. A thermal model using ANSYS to simulate the thermosiphon cooling will be presented. The thermal analysis will make provisions for including the heat load generated in the coils and structures by the secondary radiation simulated using the MARS15 code. The impact of the heat loads from supports will be considered. The thermosiphon cooling scheme will also be validated using pertinent correlations.
\end{abstract}

Keywords: Thermosiphon, Mu2e Cryogenics, Production Solenoid.

\section{INTRODUCTION}

The Production Solenoid, one of the three superconducting solenoids of the Mu2e experiment is under development at Fermilab. The Production Solenoid (PS) houses the production target and functions as the source of muons by trapping the charged pions from the production target and accelerating them toward the Transport Solenoid as they decay into muons. The PS operates over an axially graded field range of $4.6 \mathrm{~T}$ to $2.5 \mathrm{~T}$ which necessitates the coil temperatures to be within $5.1 \mathrm{~K}$ with an approximate $1.5 \mathrm{~K}$ margin over the critical temperature of $6.5 \mathrm{~K}$. The PS coil assembly consists of aluminum stabilized NbTi Rutherford type cable wound and contained within an outer shell. The coils are indirectly (conductively) cooled by aluminum cooling ribs welded on the outer shell containing $4.7 \mathrm{~K}$ helium using a thermosiphon scheme. A satellite refrigerator supplies the liquid helium over a 20 meter transmission line through a feedbox which is subcooled at the feedbox to $4.7 \mathrm{~K}$ before feeding the thermosiphon inlet. The temperature gradient within the coils has been studied via finite element analysis using ANSYS 14.5.

\section{COLD MASS ASSEMBLY}

The solenoid coil comprises of an assembly of 3 coil sections encompassing 3, 2 and 2 layers of aluminum stabilized $\mathrm{NbTi}$ superconducting cables of constant inner diameter. The coils are directly wound inside a support cylinders or outer shells of high-strength 5083-O grade aluminum alloy. The three sections bolted together through the end flanges constitute the cold mass assembly [1]. Figure 1 shows the model of the cold mass assembly. The cooling tubes are welded to the outer shell and form an integral part of the cold mass assembly. The temperatures in the coils are maintained within the design limits by fast conduction of the thermal loads to the cooling tubes by means of high purity, high conductive RRR aluminum sheets that act as thermal bridges between the inner surface of the coil and the cooling tubes.

The PS cold mass assembly and the cryostat housing are located and positioned by axial and radial support systems. The support systems of the coil and thermal shield assemblies are designed to resist axial magnetic and offcenter forces as well as gravity loads. The support systems have also been optimized to minimally contribute thermal loads and accommodate thermal contraction upon cooldown of the assembly [2].

The cold mass assembly is cryostated within a vacuum vessel that has a warm bore. Thermal radiation shields line the outside and inside of the cold mass to provide an $80 \mathrm{~K}$ thermal boundary. Lining the inside of the PS warm bore is an additional heat and radiation shield designed to protect the solenoid's superconducting coils. During experimental operation a dynamic heat load is anticipated from the particle irradiation, this results in an energy deposition in the heat shield which is asymmetric with the largest depositions being near the target and collinear with the incoming proton beam direction. 


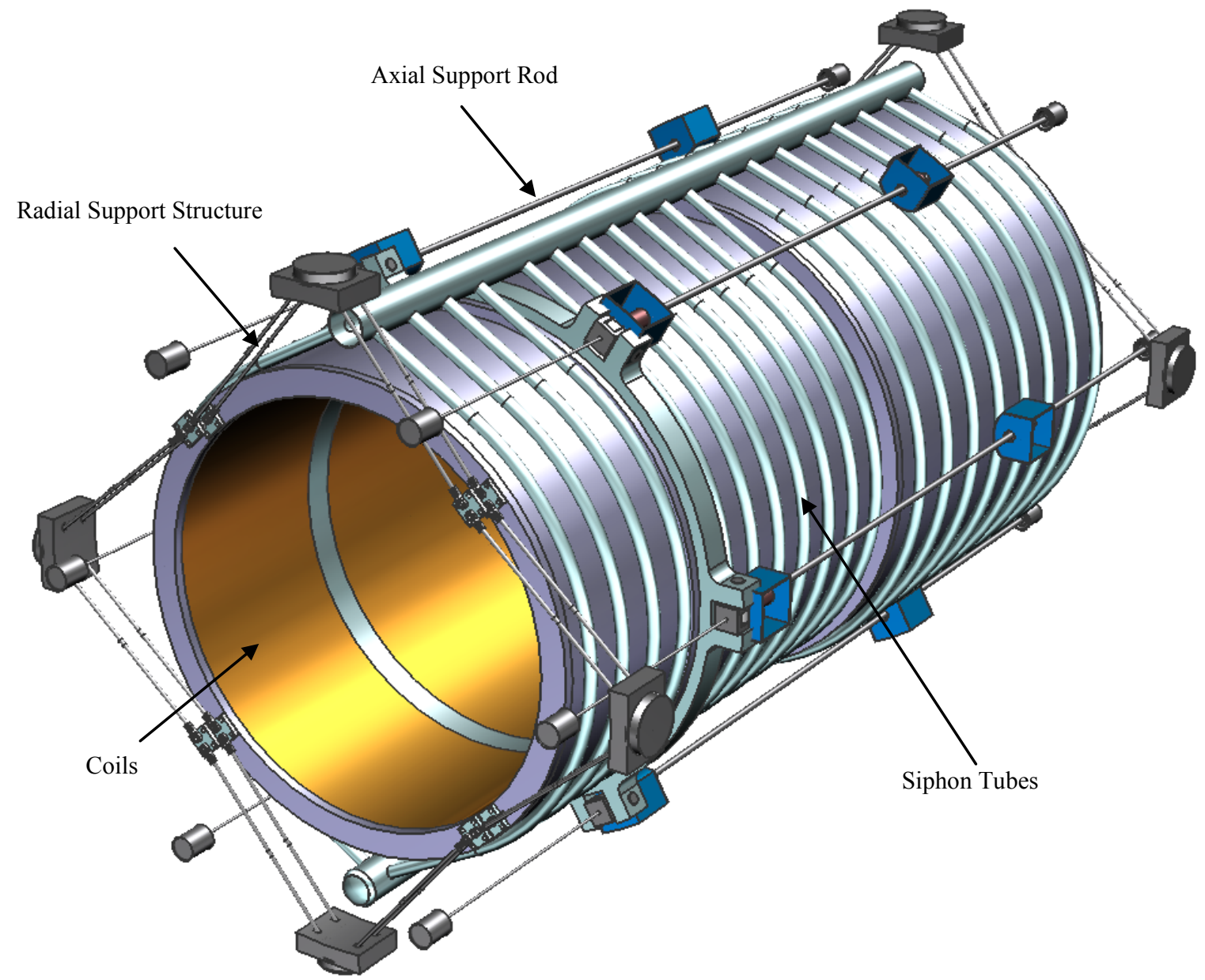

FIGURE 1. Production Solenoid Cold Mass Assembly.

\section{Thermal Loads}

The thermal loads for the PS have been conservatively estimated [2] for the standby and powered mode with no contingency. The static load includes the heat loads from the transmission lines and the feedbox and the power mode additionally includes the dynamic heat load from the particle irradiation. Table 1 shows the estimated heat loads for the PS. The dynamic heat load of the magnet is estimated based on MARS15 Monte-Carlo simulations [3].

TABLE (1). Thermal Loads for the Production Solenoid.

\begin{tabular}{|c|c|c|c|}
\hline & \multicolumn{2}{|c|}{$4.7 \mathrm{~K}$} & \multirow[t]{2}{*}{$80 \mathrm{~K}$} \\
\hline & Standby & Power & \\
\hline Transmission Line Load & $4.0 \mathrm{~W}$ & $4.0 \mathrm{~W}$ & $80 \mathrm{~W}$ \\
\hline Feedbox Heat Load & $10 \mathrm{~W}$ & $10 \mathrm{~W}$ & $60 \mathrm{~W}$ \\
\hline Magnet Load & $14.9 \mathrm{~W}$ & $64.9 \mathrm{~W}$ & $130.7 \mathrm{~W}$ \\
\hline Total Heat Load & $28.9 \mathrm{~W}$ & $78.9 \mathrm{~W}$ & $270.7 \mathrm{~W}$ \\
\hline Total Helium Flow at $4.7 \mathrm{~K}$ & $1.72 \mathrm{~g} / \mathrm{s}$ & $4.7 \mathrm{~g} / \mathrm{s}$ & - \\
\hline Total Nitrogen Flow at $80 \mathrm{~K}$ & - & - & $1.4 \mathrm{~g} / \mathrm{s}$ \\
\hline
\end{tabular}




\section{THERMOSIPHON COOLING SCHEME}

The schematic flow diagram for the PS is shown in Figure 2. Helium is supplied from the refrigerator at 3 bar, $4.8 \mathrm{~K}$ to a subcooler in the feedbox through an approximate 20 meter transmission line. The subcooler bath is maintained by dropping and boiling off a portion of the helium supply through a J-T valve and returning the vapor to the refrigerator by which a $4.6 \mathrm{~K}$ temperature is maintained in the bath. The 3 bar line is then subcooled through a heat exchanger through the bath to about $4.7 \mathrm{~K}$ and partitioned to provide three lines from the feedbox. Two of these lines are expanded though J-T valves to supply saturated helium to the magnet for cool-down, filling, warm-up and maintain liquid helium supply to the magnet thermosiphon circuit. The third line is used to conductively cool and intercept the heat load in the transmission line from the feedbox to the magnet which is about 20 meter long. The return lines from both the magnet and the transmission lines are dropped back into the subcooler.

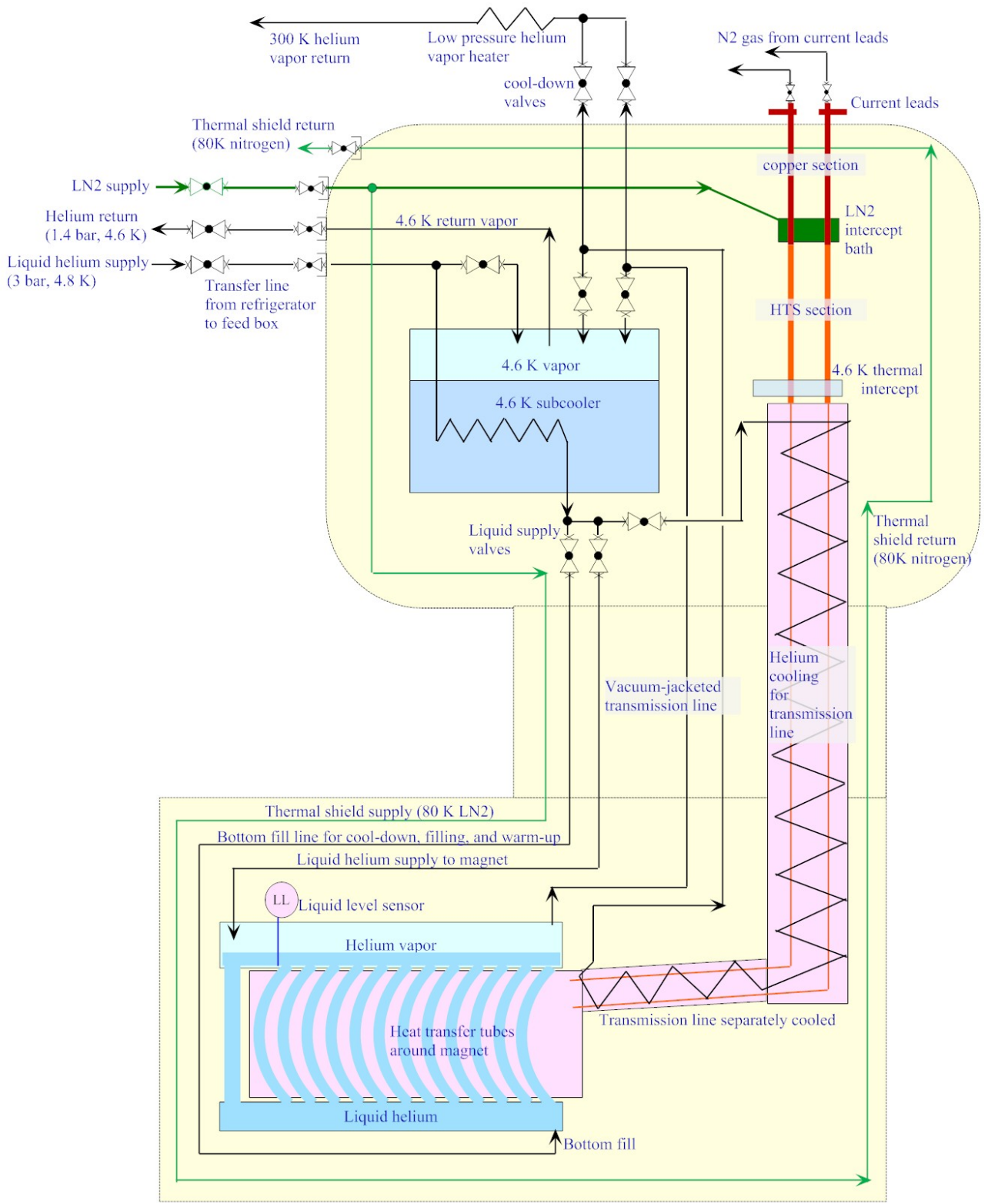

FIGURE 2. Schematic Flow Diagram for the Thermosiphon Cooling Concept. 
The thermal shield cooling is achieved by liquid nitrogen which also intercepts heat in the transmission line, distribution and feedboxes. A part of the LN2 is also used to intercept heat from the HTS portion of the current leads. The nitrogen return is just warmed and vented to the atmosphere. The cool-down of the magnet is achieved at a controlled rate by maintaining a $20 \mathrm{~K}$ difference between the helium inlet and outlet to reduce the thermal contraction stresses induced in the magnet and to maintain it below the allowable stress limits.

The thermosiphon circuit consists of array of tubes over the entire length of the magnet to provide uniform cooldown of the magnet. A redundant circuit is planned to be incorporated by connecting alternate cooling tubes to a second circuit that can be isolated using shut-off valves. There will be two sets of inlet and HGRPs (Helium Gas Return Pipes) each connected to one of the thermosiphon circuit. A redundancy is necessary to accommodate for any failure in the thermosiphon circuit and overcome the need for a few years downtime on repair of the irradiated solenoid. The total helium flow is dictated by the total heat load and is calculated to be around $4.7 \mathrm{~g} / \mathrm{sec}$.

\section{THERMAL ANALYSIS}

The thermal analysis was performed on half symmetric model of the production solenoid inclusive of all the features of insulation, thermal bridges and thermal plates designed to extract the heat loads from the coils. The details of the cable and insulation have been described in [4]. Figure 4 shows the details of the representative thermal model. The thermal bridges are made of $1 \mathrm{~mm}$ Al sheets of the same composition as the cable stabilizer and are installed on the inner and outer surfaces of the coil stack and extend along the entire length of the coils. The ends of the thermal bridges are bent 90 degrees and bonded to the thermal plates during or after the coil impregnation.

The thermal plates are made of $4 \mathrm{~mm} \mathrm{Al}$ sheets of the same material as the thermal bridges and run along the sides of the coil and are welded to identical Al strips that are pre E-beam welded to the cooling tubes. Thus the high purity Al sheet is responsible for the fast conduction of the heat loads to the cooling tubes. The thermal gradient along the radial direction is very high and has negligible contribution to the heat extraction; this was also verified by the thermal analysis.

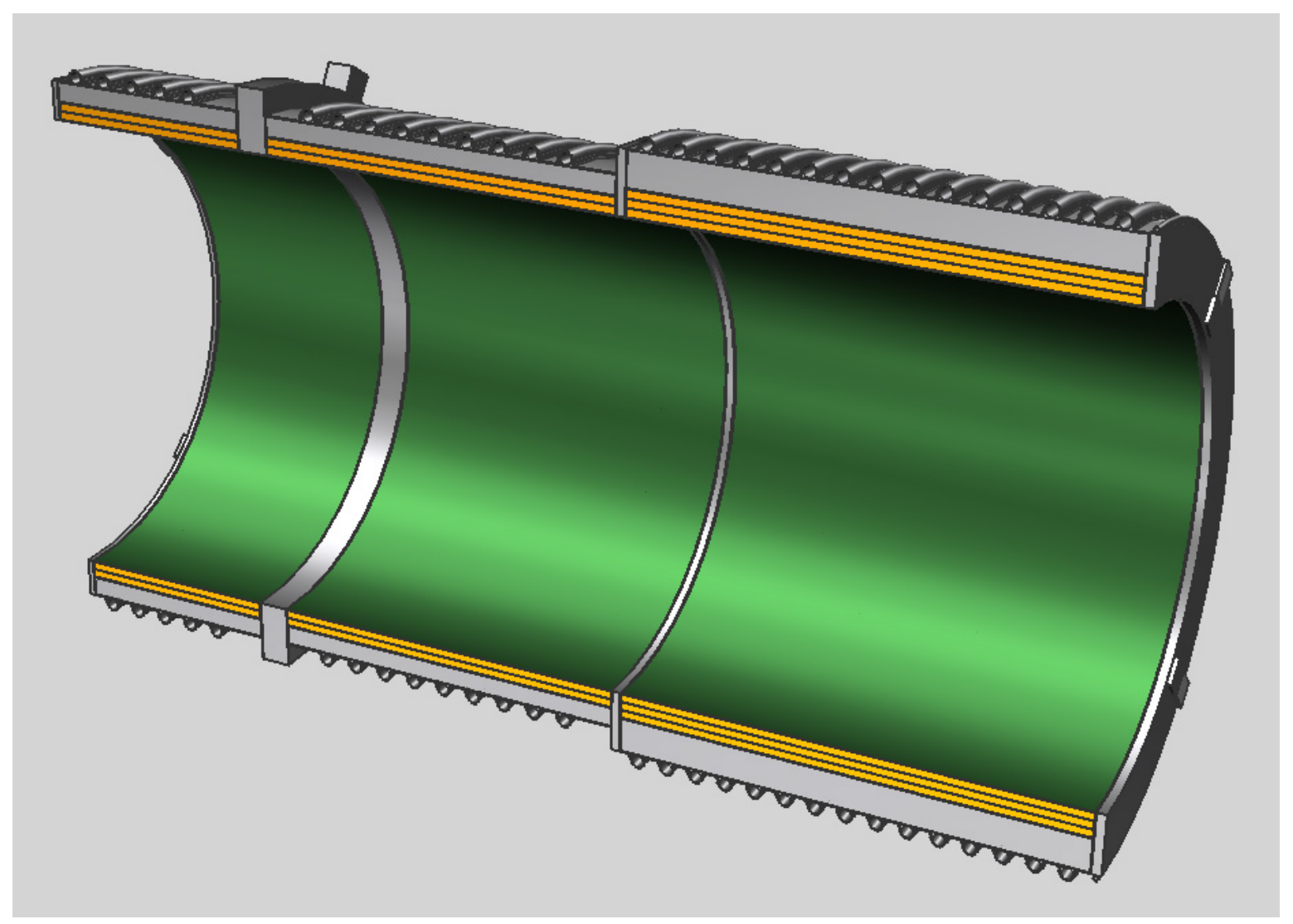

FIGURE 3. Half Model for Thermal Analysis. 


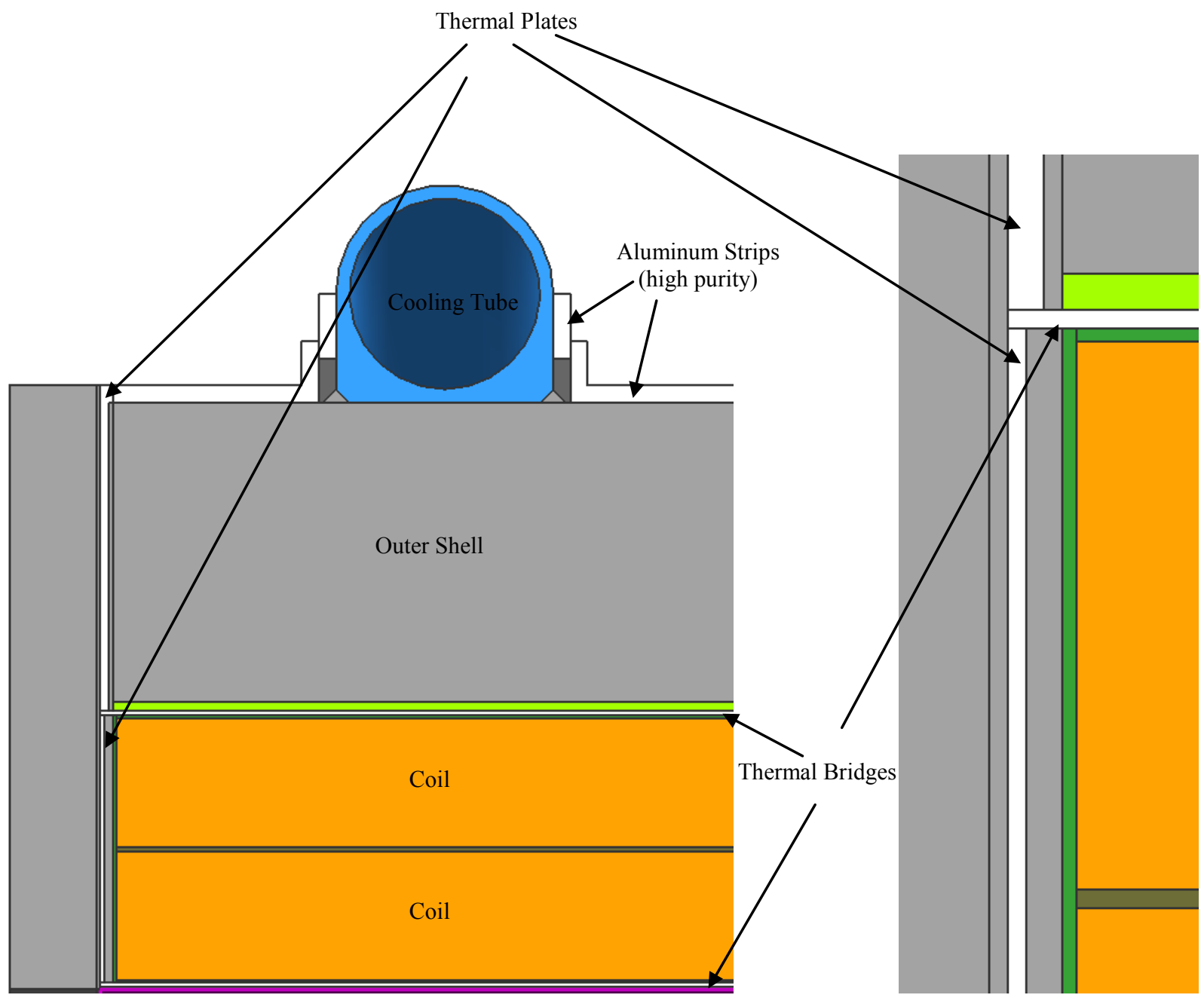

FIGURE 4. Cross Section of Coil 3 Showing the Thermal Bridges and Plates.

The ends of the thermal plates are stress-relieved by providing a clearance at the corners of the support shells to accommodate the differential contraction between the coils and the shells due to the thermal gradients and Lorentz forces. Layers of mica paper are introduced between the thermal plates, flanges and support shells to avoid accumulation of shear stresses at these interfaces [4]. The thermal plates are provided at each end of every coil assembly and connected to the cooling tubes. The tubes are in turn interconnected with high purity aluminum strips. These interconnecting high purity aluminum strips are also being planned to be used for external splice connections as they would aid the cooling of the splice joints by connecting them to the siphon tubes.

Steady state thermal analyses were performed on the PS half model to estimate the coil temperatures during operation and to ensure the temperature hikes fell within the operating limits. The thermal loads were activated for the model including the 80 kelvin radiative heat load and the dynamic heat load from the particles. The material properties were parametrically calculated for the coil stacks within due considerations to orthotropic nature of the different materials. The simulations were performed in ANSYS 14.5 Workbench with a three dimensional half model meshed with about 800,000 solid elements. The coil stacks including the intermediate insulating layers were meticulously modeled to capture the thermal gradients in the cold mass assembly. Figure 5 shows the result of the thermal analyses for the nominal MARS estimation of the dynamic heat load. As seen the temperature hike is within the established thermal margin of 5.1 kelvin, this is also true for a dynamic heat load about $15 \%$ in excess of the nominal value. This has been established to accommodate typical errors that are associated with the Monte Carlo simulations. 


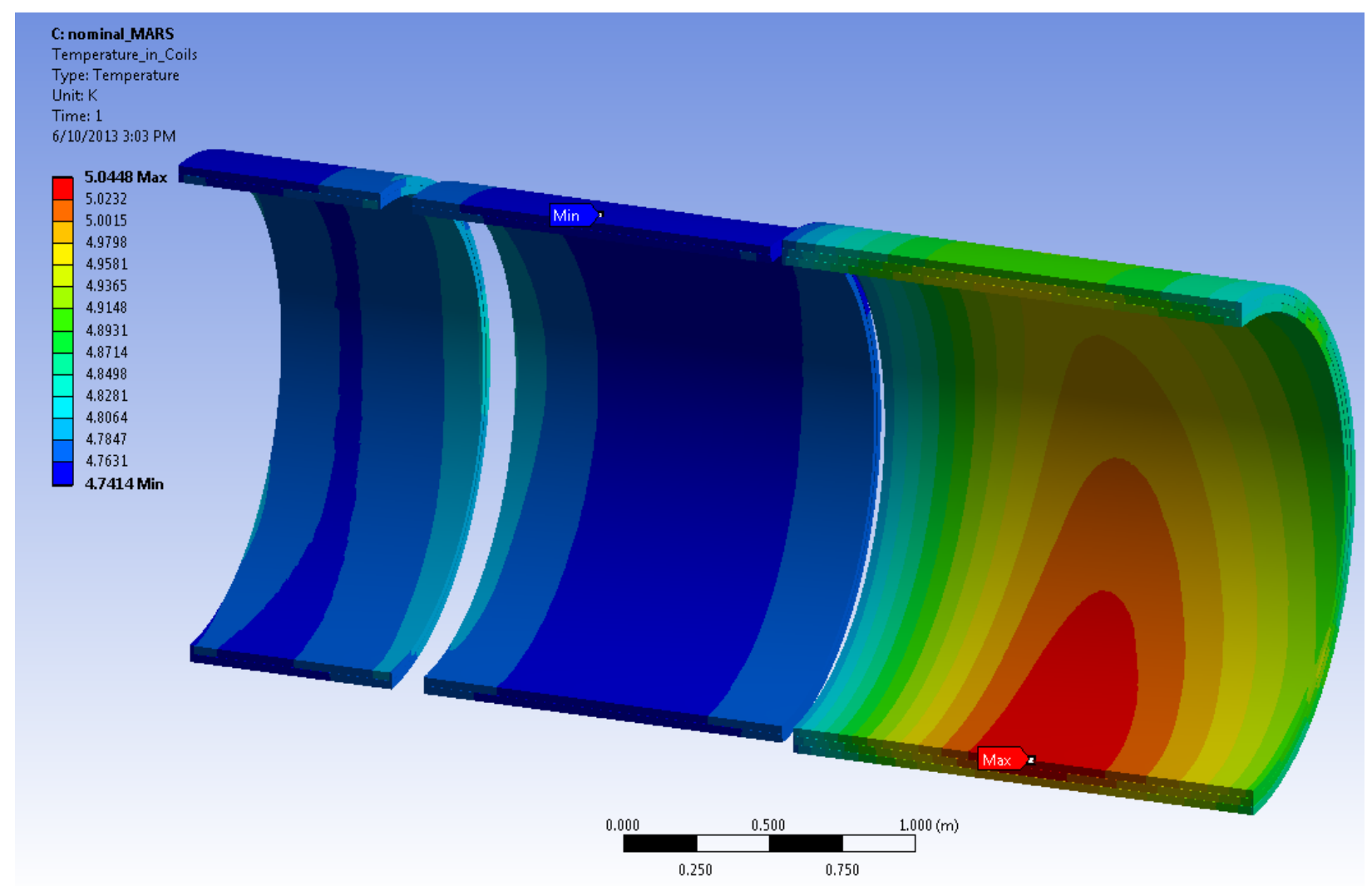

FIGURE 5. Temperature Map of the Coils.

\section{CORRELATIONS, LIMITATIONS AND VALIDATIONS}

The thermosiphon cooling scheme inherently involves a two phase flow heat transfer and some of the pertinent correlations, limitations have been studied and the design validated. A natural and fundamental concern associated with the two phase heat transfer is the ability to conduct the desired cooling within the nucleate boiling regime and avoid the regime of instabilities that can occur in the plug/slug flow. In order to characterize the flow and ensure the existence of bubbly flow, the void fraction was estimated based on the drift flux model [5]. The formulation provided by Zuber and Findlay [5] for vertical upflow is given by the following expression.

$$
\alpha=\frac{V_{g} / j}{C_{o}+u_{g j} / j}
$$

Where, $\alpha$ is the void fraction, $\mathrm{V}_{\mathrm{g}}$ is the gas superficial velocity, $\mathrm{j}$ is the average velocity, $\mathrm{u}_{\mathrm{gj}}$ is the drift velocity and $\mathrm{C}_{\mathrm{o}}$ is the distribution parameter which has a value of 1.13. The maximum void fraction corresponding to the maximum heat flux determined from the thermal analysis was calculated to be 0.204 which is less than 0.3 at which the transition to plug flow occurs, also the bubbly flow limit for vertical pipes is obtained at $20 \mathrm{~W}$ thermal losses which is almost twice more than the $10.4 \mathrm{~W}$ calculated from the thermal analysis [6].

Another associated correlation that was verified is the flooding correlation proposed by Wallis which applies to pipes with diameters less than $50 \mathrm{~mm}$ [5]. The general form of this correlation is given by equation (2).

$$
V_{l}^{* \frac{1}{2}}+V_{g}^{* \frac{1}{2}}=C
$$




$$
\begin{gathered}
V_{l}^{*}=\frac{V_{l} \rho_{l}^{\frac{1}{2}}}{\left[g d\left(\rho_{l}-\rho_{g}\right)\right]^{\frac{1}{2}}} \\
V_{g}^{*}=\frac{V_{g} \rho_{g}^{\frac{1}{2}}}{\left[g d\left(\rho_{l}-\rho_{g}\right)\right]^{\frac{1}{2}}}
\end{gathered}
$$

Where, $\mathrm{V}_{1}{ }^{*}$ and $\mathrm{V}_{\mathrm{g}}{ }^{*}$ are dimensionless liquid and gas superficial velocities given by the equations (3) and (4), the constant $\mathrm{C}$ has a value of about 0.8 . Again the application equation (2) to our case yields a value of $\mathrm{C} \sim 0.2$ which is well below the limit of 0.8 and this suggests that flooding will not occur during normal operation.

The siphon tube diameters were sized based on the above correlations and limitations and the wall thickness of the tubes were optimized to withstand pressure excursions in the event of a quench. The peak pressures were conservatively estimated assuming a closed system and an isochoric process which yields a peak pressure of about 8-10 MPa that would result in a circumferential stress of about $50 \mathrm{MPa}$ in the tubes. Thus a 5083-O aluminum alloy material was chosen for the siphon tubes with a wall thickness of $3.175 \mathrm{~mm}$.

\section{SUMMARY}

A conceptual design study of a thermosiphon system for the Production Solenoid has been performed. The solenoid is indirectly cooled by two phase helium in a thermosiphon mode. The cooling system is designed to maintain the coil temperatures within the 5.1 kelvin margin. Helium is supplied by a satellite refrigerator to the thermosiphon circuit which consists of two independent circuits to provide cooling in case of failure of one of the circuits. The design has been verified by performing thermal analysis and studying pertinent correlations and

limitations of the flow patterns. The detailed study of cool down, warm-up and quench recovery is currently being studied.

\section{ACKNOWLEDGMENTS}

The work is supported by Fermi Research Alliance, LLC; under contract No. DE-AC02-07CH11359 with the U.S. Department of Energy.

\section{REFERENCES}

1. V. Kashikhin et al., "Design Studies of Mu2e Production Solenoid", Mu2e Project Document No. Mu2e-doc-1110, http://mu2e-docdb.fnal.gov/cgi-bin/DocumentDatabase.

2. T.H. Nicol et al., "Mu2e Production Solenoid Cryostat Conceptual Design", Advances in Cryogenic Engineering, AIP Conf. Proc. 1434, 885-892 (2012).

3. V. Pronskikh, June 2013 (private communication).

4. V.V. Kashikhin et al., "Conceptual Design of the Mu2e Production Solenoid Cold Mass", Advances in Cryogenic Engineering, AIP Conf. Proc. 1434, 893-900 (2012).

5. P. B. Whalley, "Two-Phase Flow and Heat Transfer", Oxford University Press, 1996.

6. T. Niinikoski, "MICE Coupling Coil Cryostat Flooding Limit", 2010 (private communication). 